\title{
Image processing based eye detection methods a theoretical review
}

\author{
B. Vijayalaxmi ${ }^{1}$, Chavali Anuradha², Kaushik Sekaran ${ }^{3}$, Maytham N. Meqdad $^{4}$, Seifedine Kadry ${ }^{5}$ \\ ${ }^{1}$ Electronics \& Communication Engineering Department, Vignan Institute of Technology and Science, India \\ ${ }^{2}$ Department of BS\&H, Vignan Institute of Technology and Science, India \\ ${ }^{3}$ Department of Computer Science and Engineering, Vignan Institute of Technology \& Science, India \\ ${ }^{4} \mathrm{Al}$-Mustaqbal University College, Iraq \\ ${ }^{5}$ Department of Mathematics and Computer Science, Faculty of Science, Beirut Arab University, Lebanon
}

\begin{tabular}{l} 
Article Info \\
\hline Article history: \\
Received Sep 8, 2019 \\
Revised Dec 29, 2019 \\
Accepted Jan 8, 2020 \\
\hline
\end{tabular}

\section{Keywords:}

Driver behavior

Eye detection

Face detection

Fatigue

Skin

\begin{abstract}
Lately, many of the road accidents have been attributed to the driver stupor. Statistics revealed that about $32 \%$ of the drivers who met with such accidents demonstrated the symptoms of tiredness before the mishap though at varying levels. The purpose of this research paper is to revisit the various interventions that have been devised to provide for assistance to the vehicle users to avert unwarranted contingencies on the roads. The paper tries to make a sincere attempt to encapsulate the body of work that has been initiated so far in this direction. As is evident, there are numerous ways in which one can identify the fatigue of the driver, namely biotic or physiological gauges, vehicle type and more importantly the analysis of the face in terms of its alignment and other attributes.
\end{abstract}

This is an open access article under the CC BY-SA license.

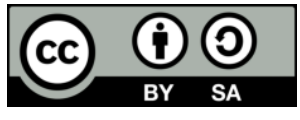

\section{Corresponding Author:}

Seifedine Kadry,

Department of Mathematics and Computer Science,

Faculty of Science,

Beirut Arab University, Lebanon.

Email: skadry@bau.edu.lb

\section{INTRODUCTION}

- Monitoring biological characteristics

Monitoring biological characterisitcs of the drivers such as electro-encephalo-gram (EEG), electro-oculo-graph (EOG), and electro-cardio-gram (ECG or EKG) that employ sensors to assess the tiredness of the person at the steering. The sleep of a person, to a large extent correlates with the functioning of the nervous system and provide useful inputs for identification of fatigue [1-3]. In this technique, the signals are captured through sensors fixed on drivers' head, chest to check EEG, ECG and the like. The rate at which the heart beats during weariness exhibits distinct variations between diverse phases of attentiveness and exhaustion. As is evident for some of the research works that demonstrate the use of HRV that aids in measurement of weariness by emitting LF and HF signals between the range of 0.04 to $0.15 \mathrm{~Hz}$ and 0.14 to $0.25 \mathrm{~Hz}$. Few research works show that drowsiness can also be measured using HRV which gives LF and HF signals, falls in range of $0.04-0.15 \mathrm{~Hz}$ and $0.14-0.25 \mathrm{~Hz}$ respectively [4].

Monitoring brain waves i.e., EEG has been a predominant instrument for assessing the tiredness of the driver. EEG waves have 4 frequency bands, delta band $(0.5-4 \mathrm{~Hz})$, which relates to sleep activity, the theta band $(4-8 \mathrm{~Hz})$, which corresponds to weariness, the alpha band $(8-13 \mathrm{~Hz})$, which indicate relaxation and creativity, and the beta band $(13-25 \mathrm{~Hz})$, which is indicative of alertness. An increase in the beta frequency band and decrease in the alpha frequency indicates drowsiness [5-11]. It has been said that the device NT-9200 for apprehending EEG signals in the varying degrees between the alert and the weary [12]. 
The physiological indicators were instrumental in measuring the fatigue of the vehicle operator. However, the presence of the electrodes all throughout was irritating posing a setback to this approach.

In yet another study, Xan Yu came up with an alternative solution to do away with the above said constraint wherein the steering of the vehicle is enclosed with a facilitating fabric to serve the purpose of electrode away from the driver. Further, the same has been replicated at the backrest of the driver's seat. Though there was a favourable implications of the ECG signals so captured but what was a matter of concern was its effect on the seatback. The most noteworthy setbacks of this were enlisted to be the need for the driver to use both his hands on the steering for the capture of the waves, the failure of this method, lest the driver uses hand gloves and the effect of noise and its interference in the working of the system.

- Tracking vehicle behaviour

The behaviour of the vehicle can be used as a parameter for assessing the weariness of the driver, given the attributes of speed, curvature, experience on the wheels, and the state of mind of the driver. The different indicators of the vehicle performance such as the reliability of the standard deviation of steering angle and its velocity [13]. In yet another similar study, put forth the use of the rate of action of the steering such as the frequency and entropy, the prerequisites ranging from very small, to minor corrections, to very frequent oscillations and deviation from the normal to the discomfort of the driver and so on [14].

The deliverables of the safety system in question can be elevated at the assessment phase, working phase as well as the grass root level of the physical vehicle state. The entire onus is on tracking the weariness degrees [15]. As is discussed earlier, the speed at which the vehicle runs, the angle of the steering and the use of the brakes and accelerator are tracked by the vehicle data. Similarly the drivers performance is assessed by keeping track of the wheel movement, alignment to the bilateral lane, changing pattern of acceleration and use of brakes and so on. The use of sensors that are incorporated o the column of the steering wheels can be resorted to for obtaining the data for understanding the steering wheel movement (SWM). As has been reported, the micro-corrections of SWM were falling within the range of 0.50 and 50 [16-19].

\section{SYSTEM BASED ON IMAGE PROCESSING}

It is an accepted fact that any system which tracks the physiological features along with the vehicle behaviour tends to be more dependable as the acquisition of the physical signals is relatively easier and reliable in terms of the results that it generates. However, what marks a drawback to otherwise very effective system is the fact that more are the number of signals, more is the difficulty in processing them thus making the system all the more complex and complicated. Another fact that cannot be undermined is the bulky size that defeats the importance of compactness for ease of installation in the vehicle. One more classification of the weariness of the vehicle operator detection system is that which is determined by the observation using image processing technique. The point to be reiterated here is that it does not make use of sensors to trace the driver signals rather tries to estimate the fatigue ness by capturing the data about the blinking of the eye.

Research in this area has time and again affirmed that the fatigue of the driver starts with its effect on his eyes and mouth. Thus, the non-intrusive systems for fatigue detection takes into account the features of the face using computer vision approach. Facial Analysis technique is commonly used for many real-time applications such as for security checks in airports, in electronic gadgets for authentication, for legal issues and more so in surveillance systems [20]. It has been agreed upon that the most frequently used face recognition tools are eigen vectors, segmentation of skin, principal component analysis, template matching and artificial neural network.

\subsection{Face detection algorithms}

Face recognition is used in multidisciplinary fields like neural networks, pattern recognition psychology and image processing. The first break through was made in the $50 \mathrm{~s}$ in the discipline of psychology. The findings drew attention towards face expression and emotional intelligence. But, the major research in this area was taken up in the 60s [21, 22]. Further, the researchers tried to extend it for human face recognition [23-26]. In extension of the same, they designed and implemented semi-automatic system. They came up with few issues that emerged up during their studies which acted as constraints for face recognition like brightness disparity, age factor, rotation and alignment of the head and face and expressions of the face, which surprisingly still exist despite of moving ahead 50 years from then $[27,28]$.

Research in 1970s led to the definition of geometric parameters and in depth study of pattern recognition in line of such parameters. To cite Kenade who attempted to design completely automated system for face recognition and found $45-75 \%$ accuracy in identification. Research in this domain continued in 1980 's wherein the advocators propounded new approaches to enhance the existing systems like template 
matching. The era of 90s mark the advent of systems relying on eigenfaces, PCA, ICA, LDA for face recognition [29].

Face detection as the name suggests involves acquisition of faces from the video stream and later is followed by extraction of regions, variations, alignment and spacing and then concludes with face recognition by involving comparison with image database [30]. In one of their studies, Yan, Kriegman a nd Ahuja attempted to classify face recognition on the basis of knowledge, feature, template and appearance. However, there is ever chance of these bases overlapping and combination of the same for developing an altogether new algorithm encompassing such categories. Enumerated below are the different categories:

- Knowledge-based methods: recognizes face based on our knowledge of human faces.

- System based on feature-invariant method: recognition of face in line with invariant method.

- Systems based on template matching: comparison of input with stored attributes.

- Systems based on appearance: identifies faces on the basis of training images.

Knowledge based methods: these rely heavily on the shape and features of the face. Along with that they may also make use of symmetric eyes and the colour of eyes being darker than the skin beneath. Similarly, the distance between eyes and the intensity of colour in the upper and the lower segment of the eyes can be adhered to. Such models are economical in terms of computation cost but may have problems with rotation and alignment. Added to it there is risk of false negatives if the rules tend to be very general. These systems work one in simpler context and find it difficult to capture complex images.

Feature-invariant methods: here, they resort to lower order attributes like corners, colour, shape and texture of the skin for detecting the face. The major drawback mentioned in the above model can be resolved here as it is rotation indifferent and scale independent. The capture time also is relatively less and hence the computation cost also comes down. However, the major setback with this is the choice of choosing the colour space, skin colour distribution model and the processing of the same [31-34].

Template matching methods: this is by far the most extensively used technique for detection as it tends to be unaffected by noise, is relatively faster and operationally more feasible that makes implementation all the more easy. This method falls back on the frontal face images that are predefined and stored in the database, with an algorithm being designed which correlates the input image with that of the stored one in the database and goes ahead with the detection. Despite of these merits, such algorithms face a limitation in terms of lighting and shapes of the face. A solution to this issue is to take an average from the face samples and store them and then to correlate the input image with the score and take it as the face position when it is the highest. This is otherwise known as the filter match method [35].

Appearance based methods: here, the onus is on statistical analysis and machine learning for detection of the required face attributes. The period from 1986 to 1990, saw the design of face recognition system by adhering to PCA whereby the face is synonymous with the coordinate system, the vectors being called eigenvectors. Further in 1997, new contributions were made in this direction based on neural network for face recognition comprising of two separate classes for face and non face attributes. However, the grave issue faced here was the training and representation of images that did not have faces. Similarly, support vector machines (SVMs) that are linear classifiers are resorted to as they maximise the margin between decision hyper plane and training set [36].

\subsection{Eye detection algorithms}

A suitable and well defined and feasible technique for eye tracking was a major challenge for the researchers in the last decade. ET scans the manoeuvre of the eyes to predict the direction in which the driver is looking and the extent of time to which he looks. ET applications cover HCI, BCI, assistive technology, e-learning, psychology and like. Later it has been explained with the movement of eye during reading and narrated that the movements are not uniform and when using mirror they are not along the phrase. Much later to this an eye monitoring device was using contact lens that would gauge the pupil direction with a hole in the lens and the aluminium pointer attached to the lens helps in tracing direction. It has been observed using mirror that eye movements are not continuously along the phrase. Later an eye tracker device has been introduced using small contact lens with a hole for pupil gaze direction reading was done using an aluminium pointer connected to lens.

It has been found with non-invasive eye tracking device, the first of its kind that relied on corneal reflection named photocronograph. Later its tried to trace horizontal movements using photographic plate and further it is designed with a photo device which recorded eye movements bilaterally [37]. The eye tracking systems encounter grave problems when the intensity of the pupil is weak and there is absence of brightness of the same. The tracing of the eye using IR lighting as the source calls for eyes that are open and unobstructed with glasses, hair or any other object, that are proximate to the camera with very less orientation under stable lighting and brightness. But such assumption are difficult to be met with as there would be constant changes in the brightness levels and blinking of eyes are but very natural and equally true 
is the movement of head. Similarly, thick lenses of the spectacles distract the IR light thus making the pupil weak. The research spoke about the pupil detection method which ignores the background and sets threshold value to the minimum possible. In extension to it, Adhoc algorithm using threshold and morphological operations for elimination of glares on the glasses was resorted to with elimination of noise [38-40].

The study carried out was found that pupil tracking using eye appearance was effective provided there was no blockage to the eyes and the eyes were open. Further it has been observed that the real time subtraction using filter to do away with the illumination issues. Despite of this, the problem of obstruction of the eye still remains [41]. It has been found that the eye tracking method by amalgamating appearance-based methods and active IR illumination approach to have the synergy of both the approaches. However, obstruction, glasses and lighting conditions still remained the constraints. The study concluded that such systems can: (i) produce quality input images (ii) combine different complementary techniques, utilizing their strengths and overcoming limitations in order to detect pupil efficiently [42].

The traditional based methods in eye detection are further classified into three categories:

- $\quad$ Template based methods

- $\quad$ Appearance based methods

- $\quad$ Feature based methods

Template based methods: during ' $90 \mathrm{~s}$, researcher used template based methods for eye detection. In this method, a generic eye shape template is created initially. Template matching using correlation is performed on input image to find eyes. Here a method has been proposed for eye detection using accurate measurement spacing using hough transform (HT). This method is time-consuming, needs high-contrast eye images and it works only on frontal images. To improve the efficiency of template based methods, it has been proposed eye detection using deformable templates. In deformable template, first the eye model is allowed to translate, rotate and deform to fit the best representation of eye shape. This method can detect eyes accurately but they are computationally expensive and require images with good contrast [43].

Later the research says that the method based on template with optical flow with the success ranging to $88 \%$ on images and $73 \%$ on TV movies. However, the demerit of this system lies in the fact that it fails under face alignment issues and takes time to read each frame. It has been observed and propounded eye detection using deformable template by minimizing energy. The period of 2006 saw many studies that put forth eye detection using binary template grouping and SVM being tested on BioID database including 23 images with a success of $96.8 \%$, the problem of obstruction and closure of eye and the brightness still remaining the same. In response to it was the detections system using binary template matching with HT with an overwhelming success of $96.6 \%$ with the limitation of failure of capturing in case of using glasses [44-46]. Another work worth mentioning is the genetic algorithm (GA) with deformable templates that are extensively fall back upon by testing on the ORL database comprising of 400 images with the rate of success being $87.2 \%$. The major merit with this is its simplicity with relatively lesser mathematical calculations and works well provided the driver does not use glasses.

Appearance based methods: as is likely from the name, such models capture eyes on the basis of photometric appearance. It calls for accumulation of huge database of training data showing diverse eye subjects, face alignment and lighting criteria. This is employed for training neural network or SVM. It has been found that the eigenvectors to categorise face, nose \& eyes. The studies continued down the line in the 1990s but mostly concentrated on frontal images [47]. The research shows attention towards eye detection with SVM classifier as the basis that was tested upon FERET databases with 450 images out of which 150 images being frontal ones and the rest being equally divided into 33.750 rotated left $\&$ poses and had an overwhelming success of $96 \%$ without obstruction of eyes .

The work says the use of eye detection based on SVM that was tested on local database with $97 \%$ success rate working under varying degrees of brightness but with a limitation of failure in capturing image in case of the driver using spectacles or face having different alignment. Moreover, the contributions propounded the utility of SVM \& Zernike moments for detection of eyes and testing of the same on ORL database of 400 images with a success of $94.6 \%$ provided there is no obstruction of the face [48]. The contribution made an attempt with neural network with HT and tested on BioID with local database images of 304 having success rate of over $98 \%$ in contrast with the earlier methods with the condition of increase in the storage capacity requirement and elevation of the computation cost. That year saw the work has been identified in the same direction whereby it was tested on Olivetti Research Lab database with 75 images therein having a rate of success of $88 \%$ despite of the varying degrees of light but is very complicated in terms of design and constraint of diverse background [49].

Feature based methods: as discussed above, this classification looks into the attributes like iris, corners, sclera in place of eyes. In this work, it has been suggested detection of the point as they found that such parameters are more static and hence easy to capture than eyes. Further the research work says that threw light on a model that enlisted six eye edges to be taken as points.Unfortunately, it did not succeed in 
detecting closed eyes or those obstructed by hair or any other thing. Further it was propounded by a method wherein the eye model was supposed to be initialized in the first frame provided there was high contrast images to scan and trace eye edges [50,51].

Similarly researchers propounded a system that relied on the vertical and horizontal projection for eye detection and concluded that the system fails to give the desired result if the eyebrows or eyelashes were not notably visible or were out of the image range and if the driver uses glasses. This takes approximately 0.6 seconds for analysing the image. Similarly, the research says that the feature extraction using nonparametric discriminant analysis (NDA) \& AdaBoost classifier that was tested on FERET database and came up with $94.5 \%$ success rate $[52,53]$. In has been identified that the technique whereby attributes like eyelid, look \& face expression are captured and exhibited $96.4 \%$ success rate. Researchers, advocated a method wherein the driver is identified using iris texture and tested on iris database CASIA with the result being a rate of success close to $98 \%$.

As an evident from the works, though there was $88 \%$ success rate of the ORL database images technique method that made use of the eye detection with SVM, yet it failed to give necessary results in case of rotated images of the driver. Another contribution in this direction has been that the technique of eye detection that necessarily incorporates the template alignment merits with the features and exhibited favourable success rate of $95.2 \%$. Yet the underlined drawback of this method was its inappropriateness if the driver used glasses [54]. As it is propounded by the analysis of the pixels of the image is costly in terms of time, effort and money when there is differences in the shape and colour of the face, they came up with a algorithm called Haar classifier to immediately pick upon objects based on Haar Feature rather than pixels.

Researchers advocated the detection of the features of the face by resorting to Haar classifier, the same being tested on FERET database. The study concluded that the detection rate to be $95 \%$ while using 1.2 GHz AMD processor. Few researchers used colour based segmentation to detect face and eyes. Similarly, the works propounded detection of the eye with the help of skin segmentation, which would otherwise be without relying on template matching and having detection rate of $98.4 \%$. The algorithm has been proposed that uses template matching where a template is created for each image depending upon the data taken from the upper half of the face. Based on this, the part comprising the eyes is cropped and is taken as template for figuring out the eyes in the input image. It witnessed a success of 78\% when tested on GTAV database [55].

Yet another study carried out the states that colour spaces can be used for face and eye detection. The said algorithm in question, works on the extraction of face by using the skin information. This algorithm tries to trace the skin region by changing RGB image to HSV which stands for Hue to give absolute colour, Saturation which talks about how white light interferes with the pure colour and Value that throws light on the intensity of the image. The algorithm demonstrated that the range of $\mathrm{H}$ lies between 0.01 and 0.1 for the skin. The algorithm witnessed a success of $95.2 \%$ when based on mathworks database images [56].

It has been identified that the utility of an algorithm that makes use of neural network perspective for eye detection by taking into consideration two phases namely the training for the same by making use of GTAV and VITS database images as well as the detection. It has been asserted that the working of this algorithm falls back upon the prerequisite of the training and the frequency of the images used for training. The more is the number the better is the outcome of the same. Two set of images are used for training the neural network are those comprising of object on one hand and the non-object images like the background, nose, eye brows etc. When tested on GTAV database, it exhibited a success of $98 \%$. However, the inhibiting factor of this model has been the fact that it gives accurate results only on straight images and is unsuccessful in tracing both the eyes in case of 900 orientation of the face. However, when the alignment of the face is by 300 to 450 it captures one eye. Another major drawback of this algorithm is its inadequate ROI that results in the eyebrows being mistakenly identified to be eyes $[57,58]$.

\section{ENCAPSULATION OF CONVENTIONAL METHODS}

Tracing of the eyes is done by resorting to noteworthy attributes like iris, colour of the pupil, shape of eye and its edges to mention a few so as to create a distinction between the eyes and other facial attributes. Unfortunately, to the dismay of the researchers, such methods don't live up to the expectations due to the constraints posed by the illumination, face alignment and obstruction and winking and closing of eyes. Though to a large extent, the brightness problem is resolved using wavelet filtering but, still it is found to be useful on in case of slight variations in brightness and does not work efficiently if otherwise.

A judicious blend of different colour conversion techniques can be employed for doing away with the illumination effects thereby leading to improved deliverables. Table 1 throws light on the diverse techniques for eye detection. The comparisons among the various eye detection techniques is done on the basis of fields, database type, frequency of images, technique involved, attainment statistics and enhancements and their constraints such as brightness, use of glasses, time consumption for capturing eyes, 
face alignment and constraints posed by backgrounds in many of the cases. Although, there are notable work carried out in this field that demonstrates the elevated success rates, yet, the implementation of such system has its own fall-backs which have been mentioned earlier. Though the amalgamation of the two different approaches i.e., feature and appearance, would lead to the elevation of the suitability, feasibility and acceptability of the eye-detection in the actual parlance, but still it calls for rigorous efforts in this direction to do away with the drawbacks of the existing eye-detection algorithms.

Table 1. Summary of eye detection techniques

\begin{tabular}{|c|c|c|c|c|c|c|}
\hline Year & Method & $\begin{array}{c}\text { Image } \\
\text { Database }\end{array}$ & $\begin{array}{l}\text { Total } \\
\text { No. of } \\
\text { images }\end{array}$ & $\begin{array}{l}\text { Success } \\
\text { Rate }(\%)\end{array}$ & Improvement & Limitations \\
\hline 1996 & $\begin{array}{l}\text { Template based } \\
\text { with optical flow }\end{array}$ & - & - & $\begin{array}{c}8873(\mathrm{TV} \\
\text { Movies) }\end{array}$ & Head Movement & $\begin{array}{l}\text { Takes more time } \\
2 \mathrm{~min} / \text { frame }\end{array}$ \\
\hline 1998 & SVM & FERET & 450 & 96 & Rotated images & Wearing Glasses \\
\hline 2000 & $\begin{array}{l}\text { Contour } \\
\text { approach }\end{array}$ & - & - & - & $\begin{array}{l}\text { Stable against blinking, head } \\
\text { translation, rotation }\end{array}$ & Takes more time \\
\hline 2002 & Edge Segment & Local & 120 & 90 & Head Movement & Wearing Glasses \\
\hline 2004 & SVM & Local & - & 97 & $\begin{array}{l}\text { Works for different } \\
\text { Illumination }\end{array}$ & $\begin{array}{l}\text { Rotated images, } \\
\text { wearing glasses }\end{array}$ \\
\hline 2006 & $\begin{array}{l}\text { Binary Template } \\
\text { matching, SVM }\end{array}$ & BioID & 23 & 96.8 & Rotated images & Eyes Closed, Reflection \\
\hline 2006 & $\begin{array}{c}\text { Binary template } \\
\text { matching \& } \\
\text { Hough } \\
\text { Transform }\end{array}$ & BioID & 23 & 95.6 & Illumination & $\begin{array}{l}\text { Rotated images, } \\
\text { wearing glasses }\end{array}$ \\
\hline 2006 & Haar Wavelets & FRGC 1.0 & - & 94.5 & Head movement & Noise misleads eyes \\
\hline 2008 & $\begin{array}{l}\text { Zernike moments } \\
\text { \& SVM }\end{array}$ & ORL & 400 & 94.6 & Head movement & Wearing glasses \\
\hline 2009 & $\begin{array}{c}\text { Deformable } \\
\text { template } \\
\text { matching }\end{array}$ & ORL & 400 & 87.2 & $\begin{array}{l}\text { Less mathematical } \\
\text { calculation }\end{array}$ & $\begin{array}{l}\text { Wearing glasses, time } \\
\text { consuming }\end{array}$ \\
\hline 2009 & $\begin{array}{l}\text { Morphology \& } \\
\text { Colour Image }\end{array}$ & - & - & 90 & Noise removal & $\begin{array}{c}\text { Fails if one or both eyes } \\
\text { are closed. } \\
15-20 \text { sec on } 2 \mathrm{GHz}\end{array}$ \\
\hline 2010 & Line edge map & Cal Tech & 240 & 91.67 & Illumination & Wearing glasses \\
\hline 2011 & $\begin{array}{c}\text { Neural Network } \\
\text { \& Hough } \\
\text { transform }\end{array}$ & $\begin{array}{l}\text { Yale, } \\
\text { BioID, } \\
\text { Local }\end{array}$ & 304 & 98.68 & Search time is reduced & $\begin{array}{c}\text { Hough Transform } \\
\text { needs large amount of } \\
\text { storage \& high cost in } \\
\text { computation }\end{array}$ \\
\hline 2011 & $\begin{array}{c}\text { Neural Network, } \\
\text { Wavelet }\end{array}$ & $\begin{array}{c}\text { Olivetti } \\
\text { research lab }\end{array}$ & 75 & 88 & $\begin{array}{l}\text { Illumination, uniform } \\
\text { background, facial } \\
\text { expression }\end{array}$ & $\begin{array}{l}\text { Complex, change in } \\
\text { background }\end{array}$ \\
\hline 2011 & $\begin{array}{c}\text { Knowledge } \\
\text { based Template }\end{array}$ & $\begin{array}{c}\text { Face } \\
\text { expression }\end{array}$ & 200 & 78 & Illumination & Closed eyes \\
\hline 2011 & $\begin{array}{c}\text { Skin } \\
\text { Segmentation }\end{array}$ & $\begin{array}{l}\text { MathWorks } \\
\text { Video }\end{array}$ & 126 & 95.2 & Rotated images & $\begin{array}{l}\text { Works only on one } \\
\text { database }\end{array}$ \\
\hline 2012 & Neural Network & GTAV & 100 & 98 & Illumination & $\begin{array}{l}\text { Head rotation, } \\
\text { background }\end{array}$ \\
\hline 2012 & $\begin{array}{l}\text { SVM \& Gabor } \\
\text { filter }\end{array}$ & $\begin{array}{l}\text { GTAV, } \\
\text { VITS }\end{array}$ & 150 & 96 & Rotation & Illumination \\
\hline
\end{tabular}

\section{CONCLUSION}

Based on the extensive review carried out on the already prevailing eye-detection techniques, it is evident that the parameters for an optimum technique can be enumerated as follows: (a) the setbacks of illumination effect can be vanquished by incorporating different colour conversion methods, (b) the synergy achieved by combination of diverse approaches would warrant a more reliable eye detection option, (c) algorithms on neural network analysis can come in handy for bringing down the search time, (d) algorithm should be capable of serving the purpose round the clock despite of the variations in the brightness, work for day/night time conditions under different brightness, clogged face and alignment, (e) there should be a provision for an alert system for cautioning the driver much in advance to avoid unwarranted accidents, (f) the complex design should be broken down into simpler ones, (g) The algorithm should be economical in terms of computation time.

Weariness of the driver has been attributed to be the most frequent reason for catastrophic road accidents worldwide, which could otherwise be avoided provided necessary interventions are made on time. This holds good for the individual drivers as well as those in the logistics industry especially those who need 
to drive for relatively very long monotonous distances without breaks. This is a socio-economic concern and can be ignored at the risk of the greater interest of the society at large.

A closer look on the data of the national highway traffic safety administration (NHTSA) drives a point home that weariness of the driver is a potential contributor for road accidents and often elevates the risk of accidents by 5-6 times as compared to watchful drivers. Added to it, a majority of the road collisions take place at speeds above the safety limits. According to the reports of the world health organization (WHO), India ranks among the top in the world in terms of pathetic road conditions and the deaths caused thereby. The extensive research in this field throws light on the fact that the tiredness and weariness of the driver is the factor that has contributed to majority of road accidents. Fatigue, on one hand effects the alertness and the response intervention and at the same time increases the probability of being engulfed in the disaster on the other hand. Added to it, the driver with sleepiness fails to respond appropriately in case of a car crash, lest he could avoid the damage.

Surprisingly, many a times, the driver may not be in a position to apprehend the degree to which he is tired and the point when he comes to saturation because of which he does not get a clue of the danger ahead. It is at this time, that the technology-enabled assistance would come handy to the driver so as to help him identify such symptoms. Such systems could be so designed to notify the driver using them in the event of loss of attention and warn them of the potential hazard awaiting them.

\section{ACKNOWLEDGEMENTS}

This paper is a part of research work on the project "Development of Non-Intrusive Driver Fatigue Detection \& Warning System to avoid on road Accidents" sanctioned under Early Career Research Award sponsored by Science \& Engineering Research Board, Govt. of India, New Delhi at Vignan Institute of Technology \& Science, Vignan Hills, Hyderabad.

\section{REFERENCES}

[1] Zhao,W., Chellappa, R., Rosenfeld, A. and Phillips, P., "Face recognition: A literature survey," ACM Computing Surveys, vol. 34, no. 4, pp.399-458, 2003.

[2] Bruner, J. S. and Tagiuri, R., "The percepton of people," Handbook of Social Psycology, vol. 2, no. 17, 1954.

[3] Lin, C. T., Wu, R. C., Liang, S. F., Chao, W. H., Chen, Y. J., and Jung, T. P., "EEG-based drowsiness estimation for safety driving using independent component analysis," IEEE Transaction on Circuits and Systems, vol. 52, pp. 2726-2738, 2005.

[4] Saito. S, "Does fatigue exist in a quantitative measurement of eye movements?," Ergonomics, vol. 35, no. 5, pp. 607-615, 1992.

[5] Shylaja S. S, Balasubramanya Murthy, K. N., Natarajan, S., Nischith, Muthuraj R, Ajay S, "Feed forward neural network based eye localization and recognition using Hough transform," International journal of advanced computer science and applications, vol. 2, no. 3, pp. 104-109, March 2011.

[6] Atallah, M. J., "Faster image template matching in the sum of the absolute value of differences measure," Image Processing, IEEE Transactions on, vol 10, pp. 659-663, 2001.

[7] Yuille, A., Hallinan,A., Cohen, D., "Feature extraction from faces using deformable templates", International Journal of Computer Vision, vol. 8, no. 2, pp. 99-111, 1992.

[8] Lingmin Meng and Nguyen,T.Q., "Frontal face localization using linear discriminant," Conference Record of the Thirty-Third Asilomar Conference on Signals, Systems, and Computers, vol. 1, pp. 745-749, 1999.

[9] Calcagnini, G., Biancalana, G., Giubilei, F., Strano, S. and Cerutti, S., "Spectral analusis of heart rate variability signal during sleep stages," Proceedings of the 16th Annual International Conference of the IEEE Engineering in Medicine and Biology Society, vol. 2, pp. 1252-1253, 1994.

[10] Shou-Der Wei and Shang-Hong Lai, "Fast Template Matching Based on Normalized Cross Correlation With Adaptive Multilevel Winner Update," IEEE Transactions on Image Processing, vol. 17, pp. 2227-2235, 2008.

[11] Bledsoe, W. W., "The model method in facial recognition,". Technical report pri 15, Panoramic Research, Inc., Palo Alto, California, 1964.

[12] Wylie, C., Shultz, T., Miller, J., Mitler, M., Mackie, R., "Commercial Motor Vehicle Driver Fatigue and Alertness Study: Technical Summary," The National Academies of Sciences, Engineering, and Medicine, 1996.

[13] Tsunoda, M., Endo, T., Hashimoto, S., Honma, S., and Honma, K., I., "Effects of light and sleep stages on heart rate variability in humans," Psychiatry and Clinical Neurosciences, vol. 55, pp. 285-286, 2001.

[14] Feng, G. C., Yuen, P. C., "Variance projection function and its application to eye detection for human face recognition," International Journal of Computer Vision, vol. 19, no. 9, pp. 899-906, 1998.

[15] Yang, M. H., Kriegman, D. J., and Ahuja, N., "Detecting faces in images: A survey," IEEE Transactions on Pattern Analysis and Machine Intelligence, vol. 24, no. 1, pp. 34-58, January 2002.

[16] Kirby,M., and Sirovich,L., "Application of the karhunen-loeve procedure for the characterization of human faces," IEEE Transactions on Pattern Analysis and Machine Intelligence, vol. 12, no. 1, pp. 103-108, 1990.

[17] Sahyadehas, A., Sundaraj, K. and Murugappan, M., "Detecting Driver Drowsiness based on Sensors: A Review," MDPI, vol. 12, no. 12, pp. 16937-16953, 2012. 
[18] Jackson, P., Hilditch, C., Holmes, A., Reed, N., Merat, N. \& Smith, L., "Fatigue and Road Safety: A Critical Analysis of Recent Evidence," Road Safety Web Department for Transport, Publication no. 21, 2011.

[19] João Dallyson Sousa de Almeida, Aristófanes Corrêa Silva, Anselmo Cardoso Paiva, "Automatic Eye Detection Using Semivariogram Function and Support Vector Machine," 17th International Conference on Systems, Signals and Image Processing,2010.

[20] Eoh, H. J., Chung, M. K, and Kim, S. H., "Electroencephalographic study of drowsiness in simulated driven with sleep deprivation," International Journal of Industrial Ergonomics, vol. 35, pp. 307-320, 2005.

[21] Toscani, L., Gangemi, P. F., Parigi, A., Silipo, R., Ragghianti, P., Sirabella, E., Morelli, M.,Bagnoli, L., Vergassola, R., and Zaccara, G., "Human heart rate variability and sleep stages," The Italian Journal of Neurological Sciences, vol. 17, pp. 437-439, 1996.

[22] Akin M., Kurt M., Sezgin N., Bayram M, "Estimating vigilance level by using EEG and EMG signals", Neural Comput. Applications, vol. 17, pp. 227-236, 2008.

[23] S. Kawato and J. Ohya, "Real-time detection of nodding and head-shaking by directly detecting and tracking the "between-eyes"," Proceedings Fourth IEEE International Conference on Automatic Face and Gesture Recognition (Cat. No. PR00580), Grenoble, France, pp. 40-45, 2000.

[24] Vanoli, E., Adamson, P. B., Lin, B., Pinna, G. D., Lazzara, R., and Orr, W. C., "Heart Rate Variability During Specific Sleep Stages-A Comparison of Healthy Subjects With Patients After Myocardial Infarction," Circulation, vol. 91, pp. 1918-1922, 1995.

[25] Qiong Wang, Jingyu Yang, "Eye detection in facial images with unconstrained background", Journal of pattern recognition research1, pp.55-62, 2006.

[26] Hyoung-Joon Kim and Whoi-Yul Kim, "Eye detection in facial images using Zernike moments with SVM," ETRI journal, vol. 30, no. 2, pp. 335-337, April 2008.

[27] Martin Gallagher, "Development of a driver alert system for road safety”, in 2006.

[28] Kenade, T., "Picture Processing System by Computer Complex and Recognition of Human Faces," PhD thesis, Kyoto University, November 1973.

[29] Mohammed Saaidia, Sylvie Lelandais, "Face detection by neural network trained with Zernike moments", Vincent Vigneron Proceedings of the 6th WSEAS International Conference on Signal Processing, Robotics and Automation, Corfu Island, Greece, pp. 16-19, 2007.

[30] Black, M. J., Yacoob, Y., "Recognizing Facial Expressions in Image Sequences Using Local Parameterized Models of Image Motion”, International Journal of Computer Vision, vol. 25, no. 1, October, pp. 23-48, 1997.

[31] Tian, Y., Kanade, T., Cohn, J. F., "Dual-state parametric eye tracking," Proceedings of the 4th IEEE International Conference on Automatic Face and Gesture Recognition, 2000.

[32] Yang, M. H., Ahuja, N., "Gaussian Mixture model for human skin colour and its application in image and video databases", Proceedings of SPIE: Conference on Storage and Retrieval for Image and Video Databases, vol. 3656, pp. 458-466, 1999.

[33] Ebisawa, Y., "Improved video-based eye-gaze detection method", IEEE Transcations on Instrumentation and Measurement, vol. 47, no. 2, pp.948-955, 1998.

[34] Hawlader Abdullah Al-Mamun, Nadim Jahangir, Md. Shahedul Islam and Md. Ashraful Islam, "Eye Detection in Facial Image by Genetic Algorithm Driven Deformable Template Matching", IJCSNS International Journal of Computer Science and Network Security, vol. 9, August 2009.

[35] Mihir Jain, Suman Mitra,K ., Naresh Jotwani, D., "Eye detection using line edge MAP template," In Proceedings of the Third International Conference on Computer Vision Theory and Applications, PP. 152-157, 2008

[36] A. G. Daimer, “The Electronic Drawbar," June 2001. [Online]. Available: http://www.daimler.com

[37] Ji, Q., Zhu, Z., "Eye and gaze tracking for interactive graphic display", 2nd International Symposium on Smart Graphics, Hawthorne, NY, USA, 2002.

[38] Kun Peng, Liming Chen, Su Ruan and Georgy Kukharev, "A Robust and Efficient Algorithm for Eye Detection on Gray Intensity Face", International Conference on Pattern Recognition and Image Analysis, vol. 3687, pp. 302-308, 2005.

[39] Jeffrey Huang, David Lie, Xuhui Shao, Harry Wechsler, "Pose Discrimination and Eye Detection using SVM," Proceeding of NATO-ASI on Face Recognition: From Theory to Applications, 1998.

[40] Feng, G. C., Yuen, P. C., "Multi-cues eye detection on gray intensity image," Pattern recognition, vol. 34, pp.1033-1046, 2001.

[41] V. Laxmi and P. S. Rao, "Eye detection using Gabor Filter and SVM," 2012 12th International Conference on Intelligent Systems Design and Applications (ISDA), Kochi, pp. 880-883, 2012.

[42] Vijayalaxmi, Sreehari, "Knowledge based template for Eye detection", National Conference on Microwave, Antenna \& Signal Processing, pp. 90, April 2011.

[43] Vijayalaxmi, P.Sudhakar, Sreehari, "Neural Network Approach for eye detection",The Second International Conference on Computer Science, Engineering and Applications (CCSEA-2012), May 26-27, 2012.

[44] Haro,A., Flickner,M., Essa,I., "Detecting and tracking eyes by using their physiological properties, dynamics, and appearance," Proceedings IEEE CVPR 2000, Hilton Head Island, South Carolina, 2000.

[45] Sekaran, K., \& Krishna, P. V., "Big Cloud: a hybrid cloud model for secure data storage through cloud space," International Journal of Advanced Intelligence Paradigms, vol. 8, no. 2, pp. 229-241, 2016.

[46] Sekaran, Kaushik, Mohammad S. Khan, Rizwan Patan, Amir H. Gandomi, Parimala Venkata Krishna, and Suresh Kallam, "Improving the response time of m-learning and cloud computing environments using a dominant firefly approach," IEEE Access vol. 7, pp. 30203-30212, 2019. 
[47] Sekaran, Kaushik, P. Chandana, N. Murali Krishna, and Seifedine Kadry. "Deep learning convolutional neural network (CNN) With Gaussian mixture model for predicting pancreatic cancer." Multimedia Tools and Applications, pp. 1-15, 2019.

[48] Phillip Ian Wilson, Dr. John Fernandez, "Facial feature detection using haar classifiers", JCSC, vol. 21, no. 4, April 2006.

[49] Hsu, R. L., Abdel-Mottaleb, M., Jain, A. K., "Face detection in colour images", IEEE Transaction Pattern Analysis Machine Intelligence, vol. 24, no. 5, pp. 696-706, 2002.

[50] Neeta Parmar, "Drowsy Driver Detection System", Design project, Department of Electrical and Computer Engineering, Ryerson University, (2002).

[51] M. C. Shin, K. I. Chang and L. V. Tsap, "Does colorspace transformation make any difference on skin detection?," Sixth IEEE Workshop on Applications of Computer Vision, 2002. (WACV 2002). Proceedings., Orlando, FL, USA, pp. 275-279, 2002.

[52] Phung, S. L., Bouzerdoum, A., Chai, D., "Skin segmentation using colour pixel classification: analysis and comparison", IEEE Transaction Pattern Analysis Machine Intelligence, vol. 27, no. 1, 2005.

[53] P. Viola and M. Jones, "Rapid object detection using a boosted cascade of simple features," Proceedings of the 2001 IEEE Computer Society Conference on Computer Vision and Pattern Recognition. CVPR 2001, Kauai, HI, USA, pp. I-I, 2001.

[54] Karamjeet Singh, Rupinder Kaur,"Physical and Physiological Drowsiness Detection Methods", International Journal of IT, Engineering and Applied Sciences Research, vol. 2, pp.35-43, 2013.

[55] Lal, S. K. L., Craig, A., Boord, P., Kirkup, L., and Nguyen, H., "Development of an algorithm for an EEG-based driver fatigue countermeasure," Journal of Safety Research, vol. 34, pp. 321-328, 2003.

[56] Greenspan,H., Goldberger,J., Eshet, I., "Mixture model for facecolor modeling and segmentation", Pattern Recognition Lett., vol. 22, no. 14, pp. 1525-1536, 2001.

[57] Zhang Zhengzhen and Shi Yuexiang, "Skin color detecting unite YCgCb color space with YCgCr color space," 2009 International Conference on Image Analysis and Signal Processing, Taizhou, pp. 221-225, 2009.

[58] Sekaran, Kaushik, and P. Venkata Krishna. "Cross region load balancing of tasks using region-based rerouting of loads in cloud computing environment." International Journal of Advanced Intelligence Paradigms, vol. 9, no. 5-6, pp. 589-603, 2017. 